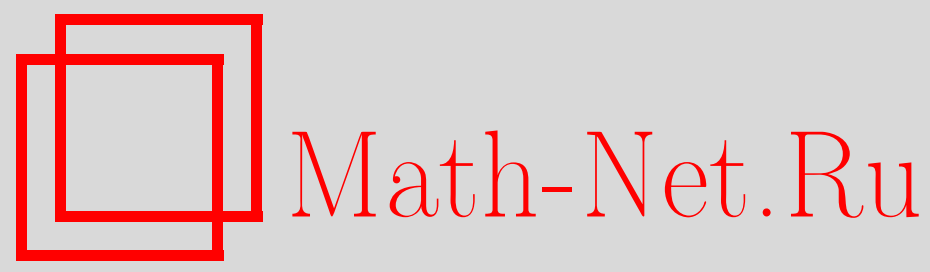

Е. Д. Нурсултанов, О мультипликаторах рядов Фурье по тригонометрической системе, Матем. заметки, 1998, том 63, выпуск 2, 235-247

DOI: https://doi.org/10.4213/mzm1270

Использование Общероссийского математического портала Math-Net.Ru подразумевает, что вы прочитали и согласны с пользовательским соглашением http://www . mathnet.ru/rus/agreement

Параметры загрузки:

IP : 54.80 .97 .219

26 апреля 2023 г., $17: 52: 46$

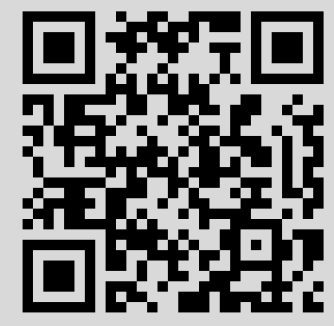




\section{О МУЛЬТИПЛИКАТОРАХ РЯДОВ ФУРЬЕ ПО ТРИГОНОМЕТРИЧЕСКОЙ СИСТЕМЕ}

\section{Е. Д. Нурсултанов}

В работе доказаны теоремы о муль типликаторах рядов Фурье в $L_{p}$, где условия зависят от параметра $p$. Построен пример, показывающий существенность этих условий.

Библиографоия: 13 названий.

Введение. Пусть $f(x)$ - функция из $L_{p}\left(\mathbb{T}^{n}\right)$,

$$
\sum_{k \in \mathbb{Z}^{n}} \widehat{f}(k) e^{i k x}
$$

- ее ряд Фурье по тригонометрической системе $\left\{e^{i k x}\right\}_{k \in \mathbb{Z}^{n}}, A$ - конечное подмножество $\mathbb{Z}^{n}=\left\{k=\left(k_{1}, \ldots, k_{n}\right): k_{i} \in \mathbb{Z}, i=1, \ldots, n\right\}$.

Пусть $\lambda=\left\{\lambda_{k}\right\}_{k \in \mathbb{Z}}$ - последовательность комплексных чисел. Будем говорить, что $\lambda \in \mathbf{m}_{p}$, т.е. $\lambda$ является мультипликатором Фурье в $L_{p}$, если для функции $f \in L_{p}\left(\mathbb{T}^{n}\right)$ с рядом Фурье (1) найдется функция $f_{\lambda} \in L_{p}\left(\mathbb{T}^{n}\right)$, ряд Фурье которой совпадает с рядом (1) и оператор $T_{\lambda} f=f_{\lambda}$ ограничен в $L_{p}\left(\mathbb{T}^{n}\right)$. Класс $\mathbf{m}_{p}$ является линейньм пространством с нормой $\|\lambda\|_{\mathbf{m}_{p}}=\left\|T_{\lambda}\right\|_{L_{p} \rightarrow L_{p}}$.

Истоком теории мультипликаторов рядов Фурье является теорема М. Рисса [1], в которой показано, что характеристическая функция $\chi_{I}$ отрезка $I$ из $\mathbb{Z}$ является мультипликатором в $L_{p}[0,2 \pi)$, причем $\left\|\chi_{I}\right\|_{\mathbf{m}_{p}} \leqslant c$, постоянная $c$ не зависит от выбора отрезка $I$.

В 1939 году Марцинкевич [2] получил следующую теорему о мультипликаторах рядов Фурье.

Теорема (Марцинкевич). Пусть $1<p<\infty$, последовательность вещественных чисел $\lambda=\left\{\lambda_{m}\right\}_{m \in \mathbb{Z}}$ удовлетворяет условию

$$
F_{0}(\lambda)=\sup _{m \in \mathbb{N}}\left(\sum_{k=2^{m}}^{2^{m+1}}\left|\lambda_{k}-\lambda_{k+1}\right|+\left|\lambda_{-k}-\lambda_{-k-1}\right|\right)+\sup _{m \in \mathbb{Z}}\left|\lambda_{m}\right|<\infty .
$$

Тогда $\lambda$ - мультипликатор в $L_{p}[0,2 \pi) u\left\|\lambda_{m}\right\|_{L_{p}} \leqslant c F_{0}(\lambda)$.

В теореме Марцинкевича условия на последовательность $\lambda$ одинаковы для всех $1<$ $p<\infty$, т.е. не зависят от параметра $p$. Возникает задача о получении достаточных условий принадлежности $\lambda$ пространству $\mathbf{m}_{p}$, существенно зависящих от параметра $p$. Эта проблема достаточно широко обсуждалась в книге И. Стейна [3].

В данной работе получены теоремы о мультипликаторах рядов Фурье, в которых условия зависят от $p$. Построен пример, показьвающий точность этих теорем по параметру $p$. 
1. Об ограниченности частичных сумм рядов Фурье. Пусть $f(x)$ - функция из $L_{p}\left(\mathbb{T}^{n}\right), A$ - конечное подмножество $\mathbb{Z}^{n}$. Тригонометрический многочлен

$$
S_{A}(f)=\sum_{k \in A} \widehat{f}(k) e^{i k x}
$$

назовем частичной суммой ряда Фурье функции $f(x)$, соответствующей множеству индексов $A$.

Если $A$ - отрезок (т.е. параллелепипед) в $\mathbb{Z}^{n}$, то, как следует из теоремы М. Рисса,

$$
\left\|S_{A}(f)\right\|_{p} \leqslant C\|f\|_{p}
$$

где $C$ зависит только от параметра $p$. Если в качестве $A$ берутся произвольные множества, постоянная $C$ в (2) зависит от геометрических свойств множества $A$, размерности $n$ и параметра $p$. Если $A$ - многоугольник в $\mathbb{Z}^{n}$, исследованию константы $C$ посвящены работы [4]-[10]. В этом пункте исследуется зависимость константы $C$ из (2) от структурных свойств множества $A$, а именно, от представления множества в виде объединения некоторых “элементарных" множеств (отрезков, гармонических отрезков).

Пусть $B=\{n, n+1, \ldots, n+l\}$ - отрезок в $\mathbb{Z}, d \in \mathbb{N}, d>l$. Множество вида $I=\bigcup_{k=0}^{N}[B+k d]=\bigcup_{k=0}^{N}\{m+k d: m \in B\}$ назовем гармоническим отрезком в $\mathbb{Z}$, а множество $J=\bigcup_{k \in \mathbb{Z}}[B+k d]-$ гармоническим интервалом в $\mathbb{Z}$. Тогда множество вида $I=I_{1} \times \cdots \times I_{n}$, где $I_{i}$ - гармонические отрезки в $\mathbb{Z}$, называется гармоническим отрезком в $\mathbb{Z}^{n}$, а множество $J=J_{1} \times \cdots \times J_{n}$, где $J_{i}$-гармонические интервалы в $\mathbb{Z}$, назьвается гармоническим интервалом в $\mathbb{Z}^{n}$.

Ясно, что всякий отрезок в $\mathbb{Z}^{n}$ является гармоническим отрезком в $\mathbb{Z}^{n}$.

Пусть $J=\bigcup_{m \in \mathbb{Z}^{n}}(B+m d)$ - гармонический интервал, $I_{k}=\bigcup_{m \in Q_{k}}(B+m d)$, $k=1,2, \ldots,-$ последовательность гармонических отрезков, соответствующих гармоническому интервалу $J$ и последовательности отрезков $Q_{k}=\left\{m \in \mathbb{Z}^{n}: 0 \leqslant\left|m_{j}\right| \leqslant k\right.$, $j=1, \ldots, n\}$. Здесь $m=\left(m_{1}, \ldots, m_{n}\right), d=\left(d_{1}, \ldots, d_{n}\right), m d=\left(m_{1} d_{1}, \ldots, m_{n} d_{n}\right)$.

Хорошо известно следующее утверждение.

Лемма 1. Пусть $d \in \mathbb{N}$. Тогда

$$
D_{m}=\frac{1}{d} \sum_{r=0}^{d-1} e^{i 2 \pi r m / d}= \begin{cases}1, & m \text { кратно } d \\ 0, & m \text { не кратно } d\end{cases}
$$

Лемма 2. Пусть $B=B_{1} \times \cdots \times B_{n}$ - отрезок в $\mathbb{Z}^{n}, Q_{k}=\left\{m \in \mathbb{Z}^{n}: 0 \leqslant\left|m_{j}\right| \leqslant k\right.$, $j=1, \ldots, n\}, k \in \mathbb{N}$,

$$
I_{k}=\bigcup_{r \in Q_{k}}(B+r d)=\bigcup_{r \in Q_{k}}\left(B_{1}+r_{1} d_{1}\right) \times \cdots \times\left(B_{n}+r_{n} d_{n}\right)
$$

- последовательность гармонических отрезков в $\mathbb{Z}^{n}$, сходящаяся $к$ гармоническому интервалу $J$.

Если

$$
f=\sum_{m \in \mathbb{Z}^{n}} \widehat{f}(m) e^{i m x} \in L_{p}[0,2 \pi]
$$


то последовательность частичных сумм

$$
S_{I_{k}}(f)=\sum_{m \in I_{k}} \widehat{f}(m) e^{i m x}
$$

сходится в $L_{p}\left(\mathbb{T}^{n}\right) \kappa$ функиии

$$
\begin{aligned}
S_{J}(x) & =\frac{1}{d} \sum_{0 \leqslant r \leqslant d-1} f\left(x+\frac{2 \pi r}{d}\right) D_{B}\left(\frac{2 \pi r}{d}\right) \\
& =\frac{1}{d_{1}} \cdots \frac{1}{d_{n}} \sum_{r_{1}=0}^{d_{1}-1} \cdots \sum_{r_{n}=0}^{d_{n}-1} f\left(x_{1}+\frac{2 \pi r_{1}}{d_{1}}, \ldots, x_{n}+\frac{2 \pi r_{n}}{d_{n}}\right) D_{B}\left(\frac{2 \pi r_{1}}{d_{1}}, \ldots, \frac{2 \pi r_{n}}{d_{n}}\right),
\end{aligned}
$$

где $D_{B}(x)=\sum_{m \in B} e^{i m x}-$ ядро Дирихле, соответствующее отрезку $B$ из $\mathbb{Z}^{n}$.

ДокАЗАтЕльство. Заметим, что $S_{J}(x)$ принадлежит пространству $L_{p}\left(\mathbb{T}^{n}\right)$, как конечная линейная комбинация функций из $L_{p}\left(\mathbb{T}^{n}\right)$. Покажем, что ее коэффициенты Фурье совпадают с коэффициентами Фурье $\widehat{f}(m)$ функции $f(x)$ при $m \in J=\bigcup_{k=1}^{\infty} I_{k}$ и равны 0 при $m \notin J$. Имеем

$$
\begin{aligned}
S_{J}(x) & =\frac{1}{d} \sum_{0 \leqslant r \leqslant d-1} f\left(x+\frac{2 \pi r}{d}\right) D_{B}\left(\frac{2 \pi r}{d}\right) \\
& =\sum_{m \in \mathbb{Z}^{n}}\left(\frac{1}{d} \sum_{0 \leqslant r \leqslant d-1} e^{2 \pi r m / d} D_{B}\left(\frac{2 \pi r}{d}\right)\right) \widehat{f}(m) e^{i m x}=\sum_{m \in \mathbb{Z}^{n}} b_{m} \widehat{f}(m) e^{i m x} .
\end{aligned}
$$

Таким образом, необходимо показать, что

$$
b_{m}= \begin{cases}1 & \text { при } m \in J, \\ 0 & \text { при } m \notin J .\end{cases}
$$

Несложные вычисления дают

$$
\begin{aligned}
b_{m} & =\frac{1}{d} \sum_{0 \leqslant r<d} e^{2 \pi r m / d} \sum_{k \in B} e^{-i 2 \pi r k / d}=\sum_{k \in B} \frac{1}{d} \sum_{0 \leqslant r<d} e^{i 2 \pi r(m-k) / d} \\
& =\prod_{j=1}^{n} \frac{1}{d_{j}} \sum_{k_{j} \in B_{j}} \sum_{r_{j}=0}^{d_{j}} e^{i 2 \pi r_{j}\left(m_{j}-k_{j}\right) / d_{j}}
\end{aligned}
$$

Заметим теперь, что $m_{j}-k_{j}$ может быть кратно числу $d_{j}$ лишь при одном $k_{j}^{0} \in B_{j}$, так как $\left|B_{j}\right|<d_{j}$. Таким образом, если найдется $k_{j}^{0} \in B_{j}$ такое, что $m_{j}-k_{j}^{0}$ будет кратно $d_{j}$, то по лемме 1

$$
\frac{1}{d_{j}} \sum_{r_{j}=0}^{d_{j}} e^{i 2 \pi r_{j}\left(m_{j}-k_{j}^{0}\right) / d_{j}}=1 .
$$

Но кратность $m_{j}-k_{j}^{0}$ числу $d$ при $k_{j}^{0} \in B_{j}$ означает представление $m_{j} \in k_{j}^{0}+s d_{j}, s \in \mathbb{Z}$. Это, в свою очередь, может произойти лишь в случае $m_{j} \in \bigcup_{s \in \mathbb{Z}}^{\infty}\left(B_{j}+s d_{j}\right)=\bigcup_{k=1}^{\infty} I_{k}^{j}$. 
Если же $m_{j} \notin \bigcup_{k=1}^{\infty} I_{k}^{j}$, т.е. $m_{j}-k_{j}$ не кратно $d$ ни при каком $k_{j} \in B_{j}$, то согласно лемме 1

$$
\frac{1}{d_{j}} \sum_{r_{j}=0}^{d_{j}} e^{i 2 \pi r_{j}\left(m_{j}-k_{j}^{0}\right) / d_{j}}=0 .
$$

Это доказьвает формулу (4), поскольку

$$
\bigcup_{k=1}^{\infty} I_{k}^{1} \times \cdots \times \bigcup_{k=1}^{\infty} I_{k}^{n}=\bigcup_{k=1}^{\infty} I_{k}=J
$$

Следовательно, рядом Фурье функции $S_{J}(x)$ является

$$
\sum_{m \in J} \widehat{f}(m) e^{i m x}
$$

откуда и следует утверждение теоремы.

ЗАмЕчАниЕ 1. Оператор, определенньй равенством (3), является интегральной суммой, соответствующей равномерному разбиению тора $\mathbb{T}^{n}$, интеграла

$$
S_{B}(f, x)=\int_{\mathbb{T}^{n}} f(x+y) D_{B}(y) d y,
$$

являющегося, в свою очередь, частичной суммой ряда Фурье, соответствующей параллелепипеду $B \in \mathbb{Z}^{n}$.

ЗАмЕчАниЕ 2. Нетрудно убедиться (анализируя доказательство), что в неравенствах теоремы об ограниченности частичной суммы и теоремы Литтлвуда-Пэли при $1<p<2$ (см. [1], [5]) соответствующие константы ограничены числом $p^{\prime} C$, где $p^{\prime}=$ $p /(p-1)$, т.е. константы растут при $p \rightarrow 1$ не быстрее, чем $(p-1)^{-1}$. Этот факт используется нами в дальнейшем.

ЛЕмма 3. Пусть $1<p<\infty, p^{\prime}=p /(p-1), J$ - гармонический интервал. Оператор $S_{J}(f)$ из леммы 2 является оператором $(p, p)$-сильного типа и верно неравенство

$$
\left\|S_{J}(f)\right\|_{L_{p}} \leqslant p^{\prime} C\|f\|_{L_{p}}
$$

где константа $C$ не зависит от $p, J u f \in L_{p}$.

Доказательство проводится так же, как и для частичных сумм (5). Следует только следить за константами в соответствующих неравенствах (см. замечания 1 и 2).

СлЕДСТВИЕ 1. Пусть $1<p<\infty, p^{\prime}=p /(p-1), I-$ произвольный гармонический отрезок в $\mathbb{Z}^{n}, f \in L_{p}\left(\mathbb{T}^{n}\right)$. Тогда

$$
\left\|S_{I}(f)\right\|_{L_{p}} \leqslant p^{2} C\|f\|_{L_{p}\left(\mathbb{T}^{n}\right)}
$$

әде $C$ не зависит от $p, f$ и $I$. 
ДокАЗАТЕЛЬСтво. Пусть $J$ - гармонический интервал в $\mathbb{Z}^{n}$ такой, что $J \cap I=I$. Тогда найдется отрезок $Q$ в $\mathbb{Z}^{n}$ такой, что $J \cap Q=I$. Следовательно, $S_{I}(f)(x)=$ $S_{Q}\left(S_{J}(f)\right)(x)$. Поэтому, учитьвая замечание 2 , леммы 2 и 3 , имеем

$$
\left\|S_{I}(f)\right\|_{L_{p}}=\left\|S_{Q}\left(S_{J}(f)\right)\right\|_{L_{p}} \leqslant p^{\prime} C_{1}\left\|S_{J}(f)\right\| \leqslant{p^{\prime}}^{2} C_{2}\|f\|_{L_{p}} .
$$

Следствие доказано.

Пусть $\Delta_{k}=\left\{m \in \mathbb{Z}^{n}: 2^{\left|k_{j}\right|} \leqslant m_{j} \operatorname{sign} k_{j}<2^{\left|k_{j}\right|+1}, j=1, \ldots, n\right\}, k \in \mathbb{Z}^{n}$. Тогда $\mathbb{Z}^{n}$ можно представить в виде $\mathbb{Z}^{n}=\bigcup_{k \in \mathbb{Z}^{n}} \Delta_{k}$. Это представление называется двоичным разложсением решетки $\mathbb{Z}^{n}$.

Конечное множество $I$ из $\mathbb{Z}^{n}$ будем назьвать гармоническим отрезком типа Cидон $a$, если найдутся гармонический интервал $J_{0}$ и последовательность векторов $\left\{r_{k}\right\}_{k \in \mathbb{Z}^{n}}$ из $\mathbb{Z}^{n}$ такие, что для любого $k \in \mathbb{Z}^{n}$

$$
I \cap \Delta_{k}=\left(J_{0}-r_{k}\right) \cap \Delta_{k}
$$

ЗАмечАниЕ 3. Так как гармонический интервал по каждому декартову направлению имеет свой период, если для $r_{k}=\left\{r_{1}^{k}, \ldots, r_{n}^{k}\right\}$ вьполнено (6), то для векторов $\left(r_{1}^{k}+d_{1} m_{1}, \ldots, r_{n}^{k}+d_{n} m_{n}\right), m=\left(m_{1}, \ldots, m_{n}\right) \in \mathbb{Z}^{n}$ также имеет место соотношение (6).

ЗАмЕчАнИЕ 4. Любой гармонический отрезок является гармоническим отрезком типа Сидона.

Замечание 5 . Пусть $A$ - множество Сидона в $\mathbb{Z}^{n}$, т.е. $A=\left\{\left(b_{1}^{k}, \ldots, b_{n}^{k}\right)\right\}_{k=1}^{\infty} \subset \mathbb{Z}^{n}$ таково, что

$$
\left|\frac{b_{j}^{k+1}}{b_{j}^{k}}\right| \geqslant \alpha>1, \quad k=1,2, \ldots .
$$

Тогда любое конечное подмножество этого множества можно представить в виде объединения гармонических отрезков типа Сидона, причем их количество зависит только от $\alpha$. Так, если $\alpha \geqslant 2$, то любое конечное подмножество $A$ есть гармонический отрезок типа Сидона.

Множество всех гармонических отрезков типа Сидона обозначим через $S_{0}$.

Tеорема 1. Пусть $1<p<\infty, I \in S_{0}$. Ecлu $f \in L_{p}\left(\mathbb{T}^{n}\right), m o$

$$
\left\|S_{I}(f)\right\|_{L_{p}\left(\mathbb{T}^{n}\right)} \leqslant C p^{4}\|f\|_{L_{p}}
$$

где константа $C$ не зависит от $p$, гармонического отрезка $I$ и функиии $f$.

ДокАЗАтЕльство. Пусть $I$-гармонический отрезок, $\Delta_{k_{1}}, \ldots, \Delta_{k_{N}}$ - элементы двоичного разбиения $\mathbb{Z}^{n}$, с которыми $I$ имеет непустое пересечение. Тогда, учитьвая определение гармонического отрезка типа Сидона и замечание 3 , нетрудно показать, что найдутся гармонический интервал $J_{0}$, векторы с целочисленными координатами $r_{1}=$ $\left(r_{1}^{1}, \ldots, r_{n}^{1}\right), \ldots, r_{N}=\left(r_{1}^{N}, \ldots, r_{n}^{N}\right)$ такие, что

1) $S_{I}\left(f_{k_{j}}\right)=S_{J_{0}}\left(e^{i r_{j} x} f_{k_{j}}\right)$, где $S_{B}=\sum_{m \in B} \widehat{f}(m) e^{i m x}, f_{k_{j}}=S_{\Delta_{k_{j}}}(f), j=1, \ldots, N$;

2) множества $\Delta_{k_{j}}+r_{j}, j=1, \ldots, N$, являются подмножествами различных элементов двоичного разбиения пространства $\mathbb{Z}^{n}$. 
Пусть

$$
f_{0}(x)=\sum_{j=1}^{N} e^{i r_{j} x} f_{k_{j}}(x)
$$

Тогда

$$
\begin{aligned}
\left\|S_{I}(f)\right\|_{L_{p}} & =\left\|\sum_{j=1}^{N} S_{I}\left(f_{k_{j}}\right)\right\|_{L_{p}}=\left\|\sum_{j=1}^{N} S_{J_{0}}\left(e^{i r_{j} x} f_{k_{j}}\right)\right\|_{L_{p}} \\
& =\left\|S_{J_{0}}\left(\sum_{j=1}^{N} e^{i r_{j} x} f_{k_{j}}\right)\right\|_{L_{p}}=\left\|S_{J_{0}}\left(f_{0}\right)\right\|_{L_{p}} .
\end{aligned}
$$

Из леммы 3 следует, что

$$
\left\|S_{I}(f)\right\|_{L_{p}}=\left\|S_{J_{0}}\left(f_{0}\right)\right\|_{L_{p}} \leqslant c p^{2}\left\|f_{0}\right\|_{L_{p}}
$$

Учитьвая выбор векторов $r_{1}, \ldots, r_{N}$, из теоремы Литтлвуда-Пэли и замечания 2 имеем

$$
\begin{aligned}
\left\|f_{0}\right\|_{L_{p}} & \leqslant p^{\prime 3} c_{1}\left\|\left(\sum_{j=1}^{N}\left|e^{i r_{j} x} S_{\Delta_{k_{j}}}(f)\right|^{2}\right)^{1 / 2}\right\|_{L_{p}} \\
& \leqslant p^{\prime 3} c_{2}\left\|\left(\sum_{j=1}^{N}\left|S_{\Delta_{k_{j}}}(f)\right|^{2}\right)^{1 / 2}\right\|_{L_{p}} \leqslant p^{\prime 4} c\|f\|_{L_{p}},
\end{aligned}
$$

что и требовалось доказать.

Лемма 4. Пусть оператор Т представим в виде суммы линейных операторов:

$$
T=\sum_{j=1}^{N} T_{j}
$$

әде кажсдий оператор удовлетворяет следующим условиям:

а) $\left\|T_{j}\right\|_{L_{p} \rightarrow L_{p}} \leqslant{p^{\prime}}^{\alpha} D$ при $1<p \leqslant 2$, где $D$ не зависит от $p$;

б) $\|T\|_{L_{2} \rightarrow L_{2}} \leqslant D$.

Тогда при $1<p \leqslant 2$

$$
\|T f\|_{L_{p}} \leqslant c_{p} D\left(N(\ln N)^{\alpha}\right)^{\left|1 / p-1 / p^{\prime}\right|}\|f\|_{L_{p}},
$$

m.e. $\|T\|_{L_{p} \rightarrow L_{p}} \leqslant c_{p} D\left(N(\ln N)^{\alpha}\right)^{\left|1 / p-1 / p^{\prime}\right|}$. 
ДокАЗАТЕЛЬСТво. Пусть $1<p_{0} \leqslant 1+1 / 4$. Тогда из условий леммы имеем

$$
\|T f\|_{L_{p_{0}}} \leqslant \sum_{j=1}^{N}\left\|T_{j} f\right\|_{L_{p}} \leqslant D{p_{0}^{\prime}}^{\alpha} N\|f\|_{L_{p_{0}}},
$$

т.е. норма оператора $T: L_{p_{0}} \rightarrow L_{p_{0}}$ не больше чем $D p_{0}^{\prime \alpha} N$. С другой стороны, норма оператора $T: L_{2} \rightarrow L_{2}$ не больше чем $D$. Согласно методу комплексной интерполяции получаем, что $T: L_{p} \rightarrow L_{p}$, где $1 / p=(1-\theta) / p_{0}+\theta / 2$, причем верна оценка

$$
\|T\|_{L_{p} \rightarrow L_{p}} \leqslant D\left(p_{0}^{\prime \alpha} N\right)^{1-\theta} .
$$

Теперь оценим выражение $\left(p_{0}^{\prime \alpha} N\right)^{1-\theta}$, считая $1<p_{0}<5 / 4$. Имеем

$$
\left(p_{0}^{\alpha} N\right)^{1-\theta} \leqslant 16\left(x^{-\alpha} N^{1+x}\right)^{1 / p-1 / p^{\prime}},
$$

где $x=2 /\left(p^{\prime}-2\right)$. Минимизируя по $x$ из $(0,1]$, получим, что минимум достигается при $x=[(\ln N) / \alpha]^{-1}$. Поэтому

$$
\|T\|_{L_{p} \rightarrow L_{p}} \leqslant c_{p} D\left[(\ln N)^{\alpha} N\right]^{1 / p-1 / p^{\prime}} \quad \text { при } 1+\frac{\alpha}{2 \ln N+\alpha} \leqslant p \leqslant 2 .
$$

Пусть теперь $1<p \leqslant 1+\alpha /(2 \ln N+\alpha)$, следовательно, $p^{\prime} \geqslant 1+(2 \ln N) / \alpha \geqslant(2 \ln N) / \alpha$. Из неравенства (7) имеем

$$
\begin{aligned}
\|T\|_{L_{p} \rightarrow L_{p}} & \leqslant p^{\prime} N D=p^{\prime} N^{1 / p-1 / p^{\prime}} N^{2 / p^{\prime}} D \leqslant p^{\prime} N^{1 / p-1 / p^{\prime}} e^{\alpha} D \\
& \leqslant p^{\prime} N^{1 / p-1 / p^{\prime}}(e \ln N)^{\alpha\left(1 / p-1 / p^{\prime}\right)} D .
\end{aligned}
$$

Лемма доказана.

Пусть $A$ - конечное подмножество $\mathbb{Z}^{n}$. Его всегда можно представить в виде конечного объединения непересекаюшихся отрезков из $S$ :

$$
A=\bigcup_{j=1}^{l} I_{j},
$$

причем не единственным образом. Через $[A]$ обозначим наименьшее число гармонических отрезков типа Сидона, входящих в представление (8), т.е.

$$
[A]=\min \left\{l: A=\bigcup_{j=1}^{l} I_{j}, I_{j} \cap I_{i}=\varnothing \text { при } i \neq j, I_{j} \in S\right\} .
$$

Здесь минимум берется по всевозможным представлениям вида (8).

Теорема 2. Пусть $1<p<\infty, p \neq 2, A$ - конечное подмножество $\mathbb{Z}^{n}$. Тогда имеет место оченка

$$
\left\|S_{A}(f)\right\|_{L_{p}\left(\mathbb{T}^{n}\right)} \leqslant c\left([A](\ln [A])^{4}\right)^{\left|1 / p-1 / p^{\prime}\right|}\|f\|_{L_{p}\left(\mathbb{T}^{n}\right)},
$$

где с зависит только от параметра р. 
ДокАЗАТЕЛЬСтво. Пусть $I_{1}, \ldots, I_{[A]}$ - последовательность отрезков из $S$ таких, что

$$
A=\bigcup_{j=1}^{[A]} I_{j}
$$

т.е. $I_{1}, \ldots, I_{[A]}$ определяют оптимальное представление вида (8) множества $A$.

Из теоремы 1 следует, что существует константа $c$, не зависящая от функции $f$ и от чисел $j$ и $p$, такая, что для частичных сумм $S_{I_{j}}(f)$ имеет место неравенство

$$
\left\|S_{I_{j}}(f)\right\|_{L_{p}} \leqslant p^{\prime 4} c\|f\|_{L_{p}} .
$$

Из равенства Парсеваля вытекает неравенство

$$
\left\|S_{A}(f)\right\|_{L_{2}} \leqslant\|f\|_{L_{2}} .
$$

Следовательно, операторы $T_{j}=S_{I_{j}}(f), j=1, \ldots,[A]$, и $T=\sum_{j=1}^{[A]} T_{j}$ удовлетворяют условиям леммы 4 . Отсюда следует утверждение теоремы при $1<p<2$. Оставшийся случай $2<p<\infty$ вытекает из свойств симметрии оператора взятия частичной суммы.

\section{2. Мультипликаторы рядов Фурье.}

ТЕорема 3. Пусть $1<p<\infty$ и для вещественной последовательности $\lambda=$ $\left\{\lambda_{m}\right\}_{m \in \mathbb{Z}^{n}}$ выполнено

$$
F_{p}^{1}(\lambda)=\sup _{k} \sum_{r=1}^{\left|Q_{k}\right|}\left(\lambda_{r}^{*}-\lambda_{r+1}^{*}\right)\left(\left[A_{r}\right]\left(\ln \left[A_{r}\right]\right)^{4}\right)^{\left|1 / p-1 / p^{\prime}\right|}+\left|\lambda_{\left|Q_{k}\right|}^{*}\right|<\infty,
$$

где $Q_{k}=\left\{m \in \mathbb{Z}^{n}: 0 \leqslant\left|m_{j}\right| \leqslant k, j=1, \ldots, n\right\},\left\{\lambda_{r}^{*}\right\}_{r=1}^{\left|Q_{k}\right|}-$ невозрастающая перестановка (учитывающая знак) последовательности $\left\{\lambda_{m}\right\}_{m \in Q_{k}}, A_{r}=\left\{m \in Q_{k}\right.$ : $\left.\lambda_{m}>\lambda_{r}^{*}\right\}$. Тогда $\lambda \in \mathbf{m}_{p} u\|\lambda\|_{\mathbf{m}_{p}} \leqslant c_{p} F_{p}^{1}(\lambda)$.

ДокАЗАТЕЛЬСтво. Применим преобразование Абеля и неравенство Минковского:

$$
\left\|\sum_{m \in Q_{k}} \lambda_{m} a_{m} e^{i m x}\right\|_{L_{p}} \leqslant \sum_{r=1}^{\left|Q_{k}\right|}\left(\lambda_{r}^{*}-\lambda_{r+1}^{*}\right)\left\|S_{A_{r}}(f)\right\|_{L_{p}}+\lambda_{\mid Q_{k}}^{*}\left\|S_{Q_{k}}(f)\right\|_{L_{p}},
$$

где $S_{A_{r}}(f)=\sum_{m \in A_{r}} a_{m} e^{i m x}$.

Используя теорему 2 , получим

$$
\left\|\sum_{m \in Q_{k}} \lambda_{m} a_{m} e^{i m x}\right\|_{L_{p}} \leqslant c_{p}\left(\sum_{r=1}^{\left|Q_{k}\right|}\left(\lambda_{r}^{*}-\lambda_{r+1}^{*}\right)\left(\left[A_{r}\right]\left(\ln \left[A_{r}\right]\right)^{4}\right)^{\left|1 / p-1 / p^{\prime}\right|}+\left|\lambda_{\left|Q_{k}\right|}\right|\right)\|f\|_{p}
$$

Теорема доказана.

Лемма 5. Пусть $1<p<\infty u \lambda=\left\{\lambda_{m}\right\}_{m \in \mathbb{Z}}-$ последовательность комплексных чисел из $\ell_{r}$, где $1 / r=|1 / 2-1 / p|$. Тогда $\lambda \in \mathbf{m}_{p}$. 
ДокАЗАтЕльство. Пусть сначала $2<p<\infty$. Применим последовательно неравенство Харди-Литтлвуда и неравенство Гёльдера:

$$
\left\|\sum_{k=-N}^{N} \lambda_{k} a_{k} e^{i k x}\right\|_{L_{p}} \leqslant c\|\lambda a\|_{\ell_{p^{\prime}}} \leqslant c\|a\|_{\ell_{2}} \cdot\|\lambda\|_{\ell_{r}} \leqslant c\|f\|_{L_{2}} \cdot\|\lambda\|_{\ell_{r}} \leqslant c_{p}\|f\|_{L_{p}} \cdot\|\lambda\|_{\ell_{r}} .
$$

Случай $1<p \leqslant 2$ следует из соотношения $\mathbf{m}_{p}=\mathbf{m}_{p^{\prime}}$.

ТЕОрема 4. Пусть $1<p<\infty, 1 / r=|1 / p-1 / 2|$. Если последовательность комплексных чисел $\lambda=\left\{\lambda_{k}\right\}_{k=1}^{\infty}$ такова, что

$$
F_{p}^{2}(\lambda)=\inf _{\mu=\left\{\mu_{k}\right\}_{k \in \mathbb{Z}}} \sup _{m}\left(\left|\mu_{2^{m+1}}\right|+\sum_{k=2^{m}}^{2^{m+1}}\left|\mu_{k}-\mu_{k+1}\right|+\left|\lambda_{k}-\mu_{k}\right| k^{1 / r-1}\right)<\infty,
$$

$\operatorname{mo} \lambda \in \mathbf{m}_{p} u\|\lambda\|_{\mathbf{m}_{p}} \leqslant c_{p} F_{p}^{2}(\lambda)$.

ДокАЗАТЕльСтво. Пусть $\left\{\mu_{k}\right\}$ - произвольная последовательность, удовлетворяющая условию

$$
\sup _{m}\left(\sum_{k=2^{m}}^{2^{m+1}}\left|\mu_{k}-\mu_{k+1}\right|+\left|\mu_{2^{m+1}}\right|\right)<\infty .
$$

Для этой последовательности выполняется условие теоремы Марцинкевича о мультипликаторах, поэтому $\mu \in \mathbf{m}_{p}$. Так как пространство $\mathbf{m}_{p}$ линейно, $\lambda \in \mathbf{m}_{p}$ тогда и только тогда, ког да $\lambda-\mu \in \mathbf{m}_{p}$. Из леммы 5 следует, что если $\lambda-\mu \in \ell_{r}, 1 / r=|1 / p-1 / 2|$, то $\lambda-\mu \in \mathbf{m}_{p}$, откуда

$$
\sup _{m} \sum_{k=2^{m}}^{2^{m+1}}\left|\lambda_{k}-\mu_{k}\right| k^{1 / r-1}<\infty
$$

Из условия теоремы следует, что существует последовательность $\mu^{0}$, удовлетворяющая (9), такая, что

$$
\sup _{m} \sum_{k=2^{m}}^{2^{m+1}}\left|\lambda_{k}-\mu_{k}^{0}\right| k^{1 / r-1}<\infty
$$

т.е. $\lambda-\mu^{0} \in \mathbf{m}_{p}$. Поэтому $\lambda \in \mathbf{m}_{p}$. Теорема доказана.

Теорема 5. Пусть $1<p<\infty, 1 / r=|1 / p-1 / 2|$. Если последовательность действительных чисел $\lambda=\left\{\lambda_{k}\right\}_{k=1}^{\infty}$ такова, что

$F_{p}^{3}(\lambda)=\inf _{\mu}\left(\sup _{N} \sum_{k=1}^{N}\left(\mu_{k}^{*}-\mu_{k+1}^{*}\right)\left(\left[A_{k}\right]\left(\ln \left[A_{k}\right]\right)^{4}\right)^{\left|1 / p-1 / p^{\prime}\right|}+\sup _{k}\left(\left|\lambda_{k}-\mu_{k}^{*}\right|\right) k^{1 / r}\right)<\infty$ где $A_{k}=\left\{m: \mu_{m} \geqslant \mu_{k}^{*}\right\}$, mo $\lambda \in \mathbf{m}_{p} u\|\lambda\|_{\mathbf{m}_{p}} \leqslant c_{p} F_{p}^{3}(\lambda)$.

Доказательство проводится с использованием теоремы 2 и леммы 5 , аналогично доказательству теоремы 4.

Покажем, что условия на параметры в теоремах 2-5 точны. 
Лемма 6. Пусть $1<p<\infty$. Существует последовательность $\lambda_{N}=\left\{\lambda_{k}^{N}\right\}_{k \in \mathbb{Z}}$, $N=2,3, \ldots$, maкая, чmо
a) $\left\|\lambda_{N}\right\|_{\mathbf{m}_{p}} \geqslant c_{p} N^{|1 / 2-1 / p|}$
б) $F_{p}^{1}\left(\lambda_{N}\right) \leqslant c_{p} N^{|1 / 2-1 / p|}(\ln N)^{4\left|1 / p-1 / p^{\prime}\right|}$;
в) $F_{p}^{2}\left(\lambda_{N}\right) \leqslant c_{p} N^{|1 / 2-1 / p|}$
г) $F_{p}^{3}\left(\lambda_{N}\right) \leqslant c_{p} N^{|1 / 2-1 / p|}$, где $F_{p}^{1}, F_{p}^{2}, F_{p}^{3}$ - функииональ из теорем 3-5 соответственно.

ДокАЗАТЕльство. Пусть $2<p<\infty$. Случай $1<p<2$ следует из $F_{p}^{1}=F_{p^{\prime}}^{1}$, $F_{p}^{2}=F_{p^{\prime}}^{2}, F_{p}^{3}=F_{p^{\prime}}^{3}$ и $\mathbf{m}_{p}=\mathbf{m}_{p^{\prime}}$, где $p^{\prime}=p /(p-1)$.

Рассмотрим последовательность $\left\{\varepsilon_{n}\right\}$, заданную рекуррентно: $\varepsilon_{0}=1, \varepsilon_{2 k}=\varepsilon_{k}$, $\varepsilon_{2 k+1}=(-1)^{k} \varepsilon_{k}$.

Дж. Брилхарт и Л. Карлитц [11] показали, что для этой последовательности имеет место оценка

$$
\left|\sum_{m=0}^{N-1} \varepsilon_{m} e^{i m x}\right| \leqslant c N^{1 / 2}, \quad x \in[0,2 \pi) .
$$

Для построения $\lambda_{N}$ воспользуемся леммой, доказанной М. И. Дьяченко [12].

Лемма 7 [12]. Пусть $\left\{m_{k}\right\}_{k=1}^{\infty}-$ возрастающая последовательность тех натуральных чисел, для которых $\varepsilon_{m_{k}}=-1$. Тогда $m_{k+1}-m_{k} \leqslant 5$ nри $k=1, \ldots, n$.

Пусть последовательность $\left\{m_{k}\right\}$ из леммы, т.е. $\varepsilon_{m_{k}}=-1$. Рассмотрим последовательность $\lambda_{N}=\left\{\lambda_{k}\right\}_{k \in \mathbb{Z}}$, где

$$
\lambda_{k}=\left\{\begin{aligned}
-1 & \text { при } k \in\left\{m_{j}: 1 \leqslant m_{j} \leqslant N, j=1, \ldots, N\right\} \\
0 & \text { в остальных случаях. }
\end{aligned}\right.
$$

a) Согласно лемме 7

$$
n \geqslant\left|\sum_{k=1}^{n} \lambda_{k} \varepsilon_{k}\right|=\left|\sum_{1 \leqslant m_{k} \leqslant n} \varepsilon_{m_{k}}\right| \geqslant \frac{n}{5}, \quad n=1, \ldots, N
$$

следовательно,

$$
5^{-1} \leqslant n^{-1}\left|\sum_{k=1}^{n} \lambda_{k} \varepsilon_{k}\right|
$$

Тогда при $p \geqslant 2$ из [13, теорема 3$]$ следует

$$
\left\|\sum_{k=1}^{\infty} \lambda_{k} \varepsilon_{k} e^{i m_{k} x}\right\|_{p}^{p} \geqslant c \sum_{n=1}^{\infty} n^{p-2} \bar{a}_{n}^{p}, \quad \text { где } \quad \bar{a}_{n}=n^{-1} \sum_{k=1}^{n} \lambda_{k} \varepsilon_{k}
$$

следовательно,

$$
\left\|\sum_{k=1}^{\infty} \lambda_{k} \varepsilon_{k} e^{i k x}\right\|_{p} \geqslant 5^{-1} c N^{1 / p^{\prime}}
$$


Поэтому, принимая во внимание (11),

$$
\left\|\lambda_{N}\right\|_{\mathbf{m}_{p}} \geqslant \frac{\left\|\sum_{k=1}^{N} \lambda_{k} \varepsilon_{k} e^{i k x}\right\|_{p}}{\left\|\sum_{k=1}^{N} \varepsilon_{k} e^{i k x}\right\|_{p}} \geqslant c N^{|1 / 2-1 / p|} .
$$

б) Пусть $B_{N}=\left\{m_{j}: 1 \leqslant m_{j} \leqslant N\right\}$. Так как любое множество натуральных чисел из отрезка $[1, N]$ можно представить в виде объединения не более чем $2 N^{1 / 2}$ конечных арифметических прогрессий, имеем $\left[B_{N}\right] \leqslant 2 N^{1 / 2}$. Тогда

$$
\begin{aligned}
F_{p}^{1}(\lambda) & =\sum_{r=1}^{N}\left|\lambda_{r}^{*}-\lambda_{r+1}^{*}\right|\left(\left[A_{r}\right]\left(\ln \left[A_{r}\right]\right)^{4}\right)^{\left|1 / p-1 / p^{\prime}\right|} \leqslant\left(\left[B_{N}\right]\left(\ln \left[B_{N}\right]\right)^{4}\right)^{\left|1 / p-1 / p^{\prime}\right|} \\
& \leqslant c_{1}\left(N^{1 / 2}(\ln N)^{4}\right)^{\left|1 / p-1 / p^{\prime}\right|}=c_{1} N^{|1 / 2-1 / p|}(\ln N)^{4\left|1 / p-1 / p^{\prime}\right|} .
\end{aligned}
$$

в) Из определения функционала $F_{p}^{2}(\lambda)$ следует

$$
F_{p}^{2}(\lambda) \leqslant \sup _{m} \sum_{k=2^{m}}^{2^{m+1}}\left|\lambda_{k}\right| k^{1 / r-1} \leqslant \sum_{k=1}^{N}\left|\lambda_{k}\right| k^{1 / r-1} \leqslant c N^{|1 / 2-1 / p|} .
$$

г) Как и в предыдущем неравенстве, имеем

$$
F_{p}^{3}(\lambda) \leqslant \sup _{m} \sum_{k=2^{m}}^{2^{m+1}}\left|\lambda_{k}\right| k^{1 / r-1} \leqslant c N^{|1 / 2-1 / p|} .
$$

ТЕОрема 6. Пусть $2<p<\infty$. Существует последовательность $\lambda=\left\{\lambda_{m}\right\}_{m=1}^{\infty}$ такая, что

а) $\lambda \in \mathbf{m}_{p}$ и

б) $F_{p}^{1}(\lambda)<\infty$;

в) $F_{p}^{2}(\lambda)<\infty$;

г) $F_{p}^{3}(\lambda)<\infty$;

д) $F_{0}(\lambda)=\infty$.

Здесь $F_{p}^{1}, F_{p}^{2}, F_{p}^{3}$ - функциональ из теорем $3-5$, а $F_{0}$ - функционал из теоремы Мариинкевича.

ДокАЗАТЕЛЬСТво. Пусть $\lambda_{N}=\left\{\lambda_{k}^{N}\right\}_{k=1}^{\infty}, N=2,3, \ldots,-$ последовательности из леммы 6. Положим

$$
\lambda=\sum_{N=2}^{\infty} \lambda_{N}\left(N^{3 / 2-1 / p}(\ln N)^{10}\right)^{-1} .
$$

Тогда из леммы 6 с учетом полуаддитивности функционалов $F_{p}^{1}, F_{p}^{2}, F_{p}^{3}$ имеем

$$
\begin{gathered}
F_{p}^{1}(\lambda) \leqslant \sum_{N=2}^{\infty} F_{p}^{1}\left(\lambda_{N}\right)\left(N^{3 / 2-1 / p}(\ln N)^{10}\right)^{-1} \leqslant \sum_{N=2}^{\infty} \frac{1}{N(\ln N)^{10}}<\infty \\
F_{p}^{2}(\lambda) \leqslant \sum_{N=2}^{\infty} \frac{1}{N(\ln N)^{10}}<\infty, \quad F_{p}^{3}(\lambda) \leqslant \sum_{N=2}^{\infty} \frac{1}{N(\ln N)^{10}}<\infty \\
\|\lambda\|_{\mathbf{m}_{p}} \leqslant \sum_{N=2}^{\infty} \frac{1}{N(\ln N)^{10}}<\infty
\end{gathered}
$$


Покажем теперь, что для любого $\varepsilon>0\|\lambda\|_{\mathbf{m}_{p+\varepsilon}}=\infty$. Пусть $\varepsilon_{N}=\left\{\varepsilon_{k}^{N}\right\}_{k=1}^{\infty}$, где $\varepsilon_{k}^{N}$ при $k \leqslant N$ совпадают с элементами последовательности Шапиро и равны 0 при $k>N$. Рассмотрим последовательность

$$
a=\sum_{N=2}^{\infty} \varepsilon_{N}\left(N^{3 / 2}(\ln N)^{10}\right)^{-1}=\left\{\varepsilon_{k} \sum_{N=k+1}^{\infty}\left(N^{3 / 2}(\ln N)^{10}\right)^{-1}\right\}_{k=1}^{\infty}
$$

и соответствующую функцию

$$
\begin{aligned}
f_{0}(x) & =\sum_{k=1}^{\infty} a_{k} e^{i k x}=\sum_{k=1}^{\infty} \varepsilon_{k} \sum_{N=k+1}^{\infty}\left(N^{3 / 2}(\ln N)^{10}\right)^{-1} e^{i k x} \\
& =\sum_{N=2}^{\infty}\left(N^{3 / 2}(\ln N)^{10}\right)^{-1} \sum_{k=1}^{N-1} \varepsilon_{k} e^{i k x}
\end{aligned}
$$

Тогда из неравенства (10) следует, что

$$
\left\|f_{0}\right\|_{L_{p}} \leqslant \sum_{N=2}^{\infty}\left(N^{3 / 2}(\ln N)^{10}\right)^{-1}\left\|\sum_{k=1}^{N} \varepsilon_{k} e^{i k x}\right\|_{L_{p}} \leqslant c \sum_{N=2}^{\infty} \frac{1}{N(\ln N)^{10}}<\infty .
$$

С другой стороны,

$$
f_{\lambda}=\sum_{k=1}^{\infty} \lambda_{k} a_{k} e^{i k x}=\sum_{k=1}^{\infty} \sum_{N=m_{k}}^{\infty}\left(N^{3 / 2-1 / p}(\ln N)^{10}\right)^{-1} \sum_{N=m_{k}}^{\infty}\left(N^{3 / 2}(\ln N)^{10}\right)^{-1} e^{i m_{k} x} .
$$

Из $[13$, теорема 3$]$ имеем

$$
\left\|f_{\lambda}\right\|_{p+\varepsilon}^{p+\varepsilon} \geqslant \sum_{k=1}^{\infty} k^{p+\varepsilon-2} \bar{a}_{k}^{p+\varepsilon}
$$

где

$$
\begin{aligned}
\bar{a}_{k} & =\frac{1}{k} \sum_{1 \leqslant m_{k} \leqslant k} \sum_{N=m_{k}}^{\infty}\left(N^{3 / 2-1 / p}(\ln N)^{10}\right)^{-1} \sum_{N=m_{k}}^{\infty}\left(N^{3 / 2}(\ln N)^{10}\right)^{-1} \\
& \sim \frac{1}{k}\left|\sum_{1 \leqslant m_{k} \leqslant k} \frac{1}{m_{k}^{1-1 / p}\left(\ln m_{k}\right)^{20}}\right| .
\end{aligned}
$$

Из леммы 7 следует

$$
\bar{a}_{k} \sim \frac{k^{1 / p}}{k(\ln k)^{20}}=\frac{1}{k^{1-1 / p}(\ln k)^{20}}, \quad k \geqslant 2,
$$

откуда следует расходимость ряда

$$
\sum_{k=1}^{\infty} k^{p+\varepsilon-2} \bar{a}_{k}^{p+\varepsilon} \sim \sum_{k=2}^{\infty} \frac{k^{\varepsilon / p-1}}{(\ln k)^{20(p+\varepsilon)}}=\infty,
$$

что и требовалось доказать. 


\section{СПИСОК ЦИТИРОВАННОЙ ЛИТЕРАТУРЫ}

[1] Зигмунд А. Тригонометрические ряды. Т. 2. М.: Мир, 1965.

[2] Marcinkiewicz J. Sur les multiplicateurs des séries de Fourier // Studia Math. 1939. V. 8. P. 78-91.

[3] Стейн И. Сингулярные интегралы и дифференциальные свойства функций. М.: Мир, 1973.

[4] Бабенко К. И. О сходимости в среднем кратных рядов Фурье и асимптотике ядра Дирихле сферических средних. Препринт № 52. М.: ИПМ АН СССР, 1971.

[5] Алимов Ш.А., Ильин В. А., Никишин Е. М. Вопросы сходимости кратных тригонометрических рядов и спектральных разложений. 1 // УМН. 1976. Т. 31. №6. С. 28-83.

[6] Митягин Б. С. О мультипликаторах-идемпотентах в симметрических функциональных пространствах // Функцион. анализ и его прилож. 1972. Т. 6. № 3. С. 81-82.

[7] Митягин Б. С., Никишин Е. М. О расходимости спектральных разложений в среднем и почти всюду // Докл. АН СССР. 1973. Т. 212. № 3. С. 551-552.

[8] Cordoba A. Geometric Fourier Analysis // Ann. Inst. Fourier (Grenoble). 1982. V. 32. P. $215-226$.

[9] Юдин В.А. Сферические суммы рядов Фурье в $L_{p} / /$ Матем. заметки. 1989. Т. 46. № 2 . C. $145-146$.

[10] Дьяченко М. И. Нормы ядер Дирихле и некоторых других тригонометрических полиномов в пространствах $L_{p} / /$ Матем. сб. 1993. Т. 184. № 3. С. 3-20.

[11] Brillhart J., Carlitz L. Note on the Shapiro polynomials // Proc. Amer. Math. Soc. 1970. V. 25. P. 114-118.

[12] Дьяченко М.И. О сходимости двойных тригонометрических рядов и рядов Фурье с монотонными коэффицииентами // Матем. сб. 1986. Т. 129. №1. С. 55-72.

[13] Нурсултанов Е. Д. Сетевые пространства и оценки коэффициентов Фурье по тригонометрической системе // Сборник "Теория приближений и вложений функциональных пространств". Караганда, 1994. С. 121-127.

Институт прикладной математики Республики Казахстан, г. Караганда

Поступило

E-mail : nursult@ipm.karaganda.su 26.04 .96

Исправленный вариант 